\title{
An optical method of studying the shape and movement of the chest wall in recumbent patients
}

\author{
MDL MORGAN, AR GOURLAY, DM DENISON
}

From the Lung Function Unit, Brompton Hospital; the National Spinal Injuries Centre, Stoke Mandeville; and the UK Science Centre, IBM, Winchester

ABSTRACT A method of optical mapping has been developed that allows the shape and motion of the chest wall to be studied in recumbent patients. It uses a single camera at a fixed viewpoint to determine the three dimensional coordinates of the visible surface of the body, and hence to measure the volume and shape of the visible segment. Measurements on different test objects (volumes $228-7807 \mathrm{ml}$ ) in different positions suggest a reproducibility of volume measurement of $\pm 26 \mathrm{ml}$ (SD) and a maximum volume error of $150 \mathrm{ml}$. Studies of computed tomography scans in inspiration and expiration show that it should be possible to capture $97 \%$ of respiratory motion by this method. The mean difference between optical and spirometric measurements of expired volume in six subjects was 0.25 (SD 0.2) 1 . Within subject systematic errors of up to $10 \%$ were noted. The mean deviation about the regression line of optically measured expired volume on spirometric expired volume varied from 68 to $243 \mathrm{ml}$ in the six subjects (mean 131 (SD 92) $\mathrm{ml}$ ). To examine the potential of the optical technique, for rapid data acquisition it was used to construct a 24 point flow volume loop from a normal subject.

In the previous paper ( $p$ 93) we have described an optical technique for mapping the size and shape of the thoracoabdominal wall and the manner in which it changes with breathing in upright people.' The method requires the projection and interpretation of contour lines on the front and back of the torso. This paper describes the extension of that method to people who can be studied only lying down. In principle, the recumbent patient is placed in a field of light contours in such a way that the visible surface of the torso can be illuminated and filmed, by a still or video camera, from a single point of view.

\section{Method}

\section{APPARATUS}

The patient's bed is wheeled under a specially made gantry (fig 1) which supports the camera and projectors. The gantry is rigidly constructed from sections of lightweight steel tubing (Alta Structura Ltd). The dimensions are not critical, but ours has vertical pil-

Address for reprint requests: Dr MDL Morgan, Lung Function Unit, Brompton Hospital, London SW3 6HP.

Accepted 17 November 1983 lars of $2.4 \mathrm{~m}$ and a lintel of $2.8 \mathrm{~m}$. One projector (Kodak Carousel 2020 with $35 \mathrm{~mm}$ lens) is mounted on the inside of each vertical pillar. The projectors throw a pattern of horizontal black and white sheets into the space between. A single $35 \mathrm{~mm}$ still camera (Olympus OM2 with Zuiko $35 \mathrm{~mm} \mathrm{f2.8} \mathrm{lens),}$ mounted directly overhead, is joined to the projector trays and counterbalanced in such a way that the whole instrument array can move vertically as a rigid body, to provide optimum illumination and recording for any height of bed.

\section{CONTOUR PROJECTION}

The sheets of light are produced by the projection of graded line patterns from $35 \mathrm{~mm}$ transparencies. They are scaled and positioned to produce about 40 black and white interfaces (contour lines) around the visible surface of the torso. The geometry of the projection system is not critical but must be known accurately. We have the projectors $1162 \mathrm{~mm}$ from the centre of the optical reference system and align them by projecting the grid pattern on to the centre of the opposing projector lens in such a way that a specific black-white interface can be nominated as a horizontal reference plane. 


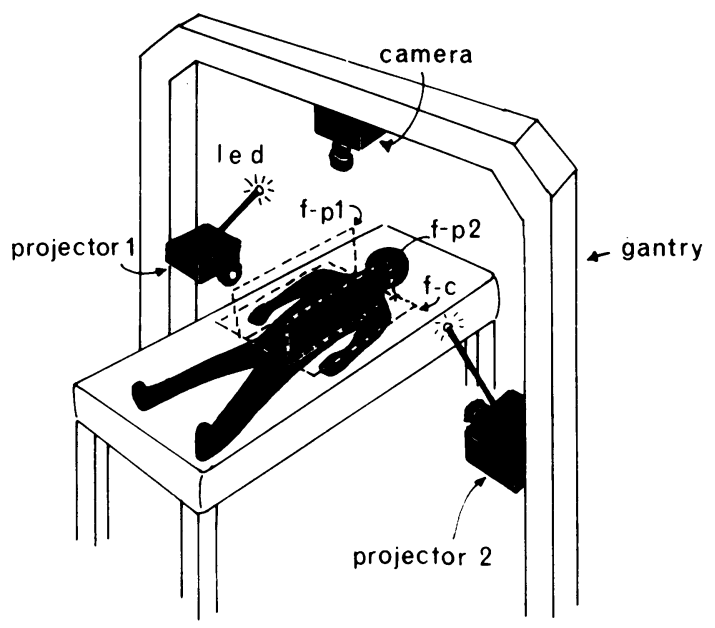

Fig 1 Schematic view of the arrangement of equipment. The camera and projectors are supported by a lightweight steel gantry. The camera is focused on the horizontal reference plane $(f-c)$ at the centre of the optical system. The projector focusing planes $f-p l$ and $f-p 2$ are $10 \mathrm{~cm}$ short of the optical centre. Light emitting diodes $(l e d)$ are fixed to the main framework as reference points.

\section{IMAGE CAPTURE}

The still camera is mounted $710.5 \mathrm{~mm}$ vertically above the centre of the horizontal reference plane. The camera and projector dimensions are the shortest that will permit illumination and recording of the whole length of the torso, given the lenses quoted. For best results the camera and projectors are focused on the planes shown in fig 1 . Two light emitting diodes are fixed to the internal frame and appear in the field of view of the camera at known positions from the optical centre, so that we can check the magnification of the image. The camera can be fired remotely by hand or automatically by, for example, a spirometer. The motor drive allows pictures to be taken at up to 4 frames a second. For some purposes it is helpful, but not essential, to have a video camera mounted on the main framework in a position as close to the still camera as will allow an unobstructed view of the patient.

\section{IMAGE PROCESSING}

To obtain quantitative data the still photographs are projected on to a digitising table, and then each contour line is identified by eye and traced by a hand held cursor. The apparent spatial coordinates of the surface of the body are entered into a computer (Prime 750) and the true spatial coordinates are calculated by correction for the divergent optics of the projectors and the convergent optics of the camera.
About 750 points are recovered from the contour lines in this way, and then the surface is reconstructed by linear or curvilinear interpolation. Computer programs can present this information in a variety of graphical and numerical forms, and also calculate whole or partial torso volumes. ${ }^{2}$

\section{Assessment of the method}

This technique recovers the shape of the visible part of the recumbent torso by a sequence of operations: projection of the grid, recording of the pattern, analogue to digital conversion, and reconstruction of the shape from digital information. The information comes from a single image of one aspect of the patient's trunk. The accuracy of these operations and the limitations of a single viewpoint were assessed as follows.

\section{PROJECTION OF THE PATTERN}

When the pattern is projected it is distorted by the surface of the body and defines its shape. It is important to be certain that the lenses of the system do not introduce distortion of their own. To check this we projected the pattern from each projector on to a vertical board at the optical centre and then at $10 \mathrm{~cm}$ in front and behind it. The position of 40 contours was marked and their distance from the optical reference plane measured in the midline and also $15 \mathrm{~cm}$ either side of it. The mean variation over the whole field of projection, from both projectors, was $0.06 \mathrm{~mm}$.

\section{CAPTURE OF THE IMAGE}

The overhead camera is also obliged to be close to the patient, and thus has a lens of short focal length that could introduce some distortion. To assess this we placed a flat and accurately known chequerboard pattern on the optical reference plane and photographed it. The image was projected on to a digitising table at known magnification and the dimensions of the sides of each of 56 squares in the field of view were compared with the measurements from the original board. The mean error in the measurement of the 20 inside squares was 0.03 (SD 0.03) $\mathrm{mm}$ and in the measurement of the 36 outside squares 0.03 $(0.02) \mathrm{mm}$. This tests the combined distortion of the camera and of projection of the image on to the digitising table.

\section{ANALOGUE TO DIGITAL CONVERSION}

In routine use, the analogue photographic image is projected on to a digitising table and the contour lines are plotted manually, introducing errors due to the projector, the table, and the operator. To examine the errors due to these three sources in 
total, we took 10 images of one model torso (a tailor's dummy) and processed the photographs individually, measuring the volume of the visible segments each time. In these circumstances we had no way of determining the actual volume of the visible segment (for example, by water displacement); but we were able to determine the reproducibility of the optical estimate, which was 7964 (26) ml (mean and SD), with a range of -44 to $+35 \mathrm{ml}$.

\section{IMAGE RECONSTRUCTION}

It is necessary to check the overall accuracy of the process by comparing the reconstructed image with the original object. With a single camera system, however, there is no back view, so such comparisons can be made only if the shape and position of the invisible part is known. We avoided this difficulty in two ways - firstly, by photographing a family of upturned pudding bowls with their rims lying in the horizontal reference plane and, secondly, by photographing the visible segment of a $30 \mathrm{~cm}$ diameter globe placed at various heights above that plane (the back boundary of this can be calculated by simple geometry). The kitchen bowls had actual volumes (measured by water displacement) that varied from 228 to $7807 \mathrm{ml}$, which were reconstructed with the volume errors shown in table 1 . The maximum discrepancy of a single estimate was $150 \mathrm{ml}(2 \%)$ for the largest bowl, but there were higher percentage errors for the very small bowls. Estimates of the volume of eight spherical segments ranging from 514 to $4131 \mathrm{ml}$ had a maximum discrepancy of $97 \mathrm{ml}(2.4 \%)$ for the largest segment (table 2). To assess the effect of changing an object's vertical position within the reference frame the volume of the same (maximum) visible segment was calculated with the sphere placed at eight different heights above the reference plane. The mean volume was $4468 \mathrm{ml}$ and the standard error $16 \mathrm{ml}$ (SD 1\%).

\section{LIMITATIONS IMPOSED BY A SINGLE FIELD OF VIEW}

When patients lie down, the area of body surface in contact with the bed is out of sight but almost stationary. Seen from a single site above, other parts of the surface close to the bed, though mobile, are also out of view because body curvature conceals them. Inevitably these two sources of uncertainty introduce some errors into the calculation of respiratory motion. To determine these we studied 95 pairs of computed tomography (CT) sections of the torso taken in full inspiration and full expiration, at different levels from the sternal notch to the iliac spine. Records from one normal subject and seven patients with common lung disorders were used. None had chest wall abnormalities. The inspiratory and
Table 1 Volumes of a set of kitchen bowls measured optically and by displacement

\begin{tabular}{lll}
\hline $\begin{array}{l}\text { Bowl } \\
\text { No }\end{array}$ & $\begin{array}{l}\text { Actual volume } \\
(\mathrm{ml})\end{array}$ & $\begin{array}{l}\text { Optical volume } \\
(\mathrm{ml})\end{array}$ \\
\hline 1 & 7807 & 7957 \\
2 & 2039 & 2069 \\
3 & 1312 & 1306 \\
4 & 1170 & 1133 \\
5 & 662 & 646 \\
6 & 408 & 463 \\
7 & 228 & 241 \\
\hline
\end{tabular}

Table 2 Comparison of optical measurements with calculated segment volumes of the visible segment of a $30 \mathrm{~cm}$ diameter globe placed at various heights above the horizontal reference plane

\begin{tabular}{lcc}
\hline Segment & $\begin{array}{l}\text { Actual volume } \\
(\mathrm{ml})\end{array}$ & $\begin{array}{l}\text { Optical volume } \\
(\mathrm{ml})\end{array}$ \\
\hline 1 & 514 & 507 \\
2 & 832 & 782 \\
3 & 1181 & 1182 \\
4 & 1629 & 1644 \\
5 & 2503 & 2513 \\
6 & 3074 & 3121 \\
7 & 3550 & 3581 \\
8 & 4131 & 4229 \\
\hline
\end{tabular}

expiratory computed tomography scans were then traced on to an exactly scaled drawing of the instrument array, and the fraction of thoracoabdominal wall visible to the camera was computed $(49.5$ (SD 1\%)). The volume recruited to the scan on inspiration was divided into seen and unseen parts, and the visible part was expressed as a percentage of the whole. It was found that $97 \%$ (range $92-105 \%$ ) of inspired volume was in the seen part-that is, could be detected by a one camera system. To discover whether there could be any benefit gained by adopting a two camera system (one camera at each angle of the gantry shown in fig 1) this graphical exercise was repeated with the same computed tomography scans superimposed on a drawing of the "new" array. The findings were almost identical ( $96 \%$ of respired motion in the "seen" part with the two cameras). Thus there was no significant advantage in this array, particularly since in practice the sensitivity of the technique increases as the angle between projector and camera approaches $90^{\circ}$.

\section{ASSESSMENT OF THE METHOD IN PATIENTS}

The measurements quoted above suggested a reproducibility of $\pm 26 \mathrm{ml}$ (SD) and an absolute volume error of $\pm 150 \mathrm{ml}$, with the ability to capture $97 \%$ of respiratory motion in subjects with normal chest walls. Many studies of breathing are not concerned with the absolute volume of the torso but with its change in size and shape. To assess this we com- 

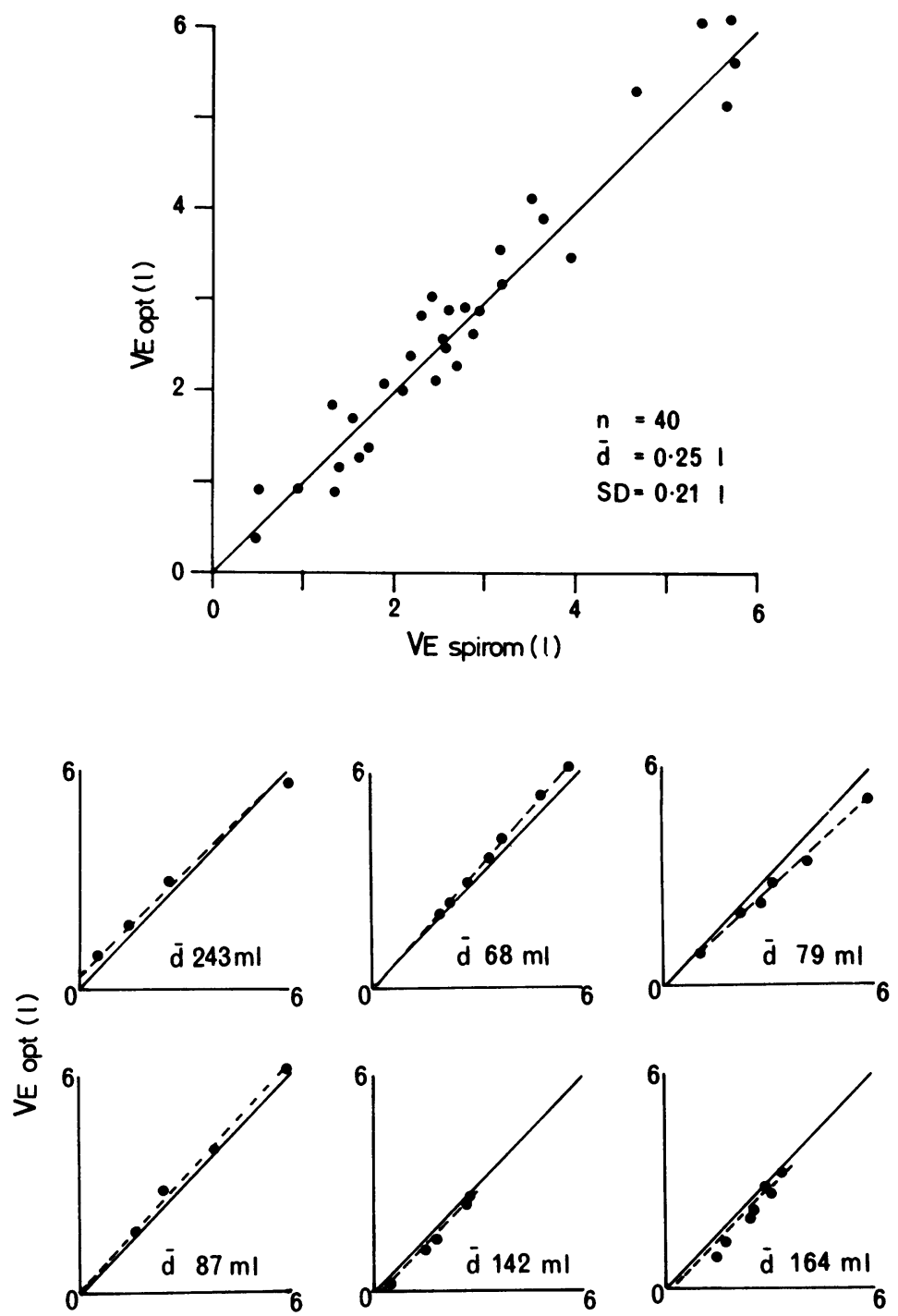

VE spirom (1)

Fig 2 (a) The combined results from six subjects of expired volume measured optically $(V E$ opt) plotted against expired volume measured with conventional spirometry (VE spirom). The mean deviation (d) from the line of identity is shown. The maximum error was $10 \%$. (b) A similar plot of expired volume for each individual. The continuous line is the line of identity and the dotted line is the regression line of VE opt on VE spirom. The mean deviation (d) from the regression line in each case is shown.

pared optical determinations of expired volume with the values obtained by conventional spirometry. Four normal and two tetraplegic subjects lay on a bed within the illuminated area and were photographed as they breathed a vital capacity into a bell spirometer. A digital display from the spirometer was visible in photographs taken at total lung capac- ity, at residual volume, and at intervals of about 1 litre between the extremes. The camera was fired automatically by the spirometer at predetermined volumes. Optical estimates of expired volume were obtained by subtraction of the instantaneous volume of the torso from its starting volume.

The results of 40 measurements show overall 
agreement. The mean difference between optical and spirometric measurements of expired volume was 0.25 (SD 0.21$) 1$, but the error of single estimates was as high as $10 \%$-that is, $600 \mathrm{ml}$ in a vital capacity of 61 (fig $2 a$ ). The physical studies quoted in the previous section suggest that an error of this magnitude cannot be accounted for by the system itself and there ought to be some other explanation. When the measurements of expired volume within each individual are examined (fig $2 b$ ) the mean deviation of observations about the regression lines for each individual is small, the mean for the six subjects being 131 (92) $\mathrm{ml}$. The origins of the systematic differences between one subject and another are not yet clear. We considered that they might be related to the degree of shadowing of the torso, but we could find no correlation between the area of the surface in shadow and the error in the optical estimate of volume change.

The temporal resolution of this technique is set by the rapidity with which the image can be captured. Currently we are limited to four frames a second with the $35 \mathrm{~mm}$ camera. To examine the potential of more rapid data acquisition we constructed an optically derived, maximal flow volume loop from a normal subject, who was chosen for his ability to perform this manoeuvre reproducibly. The camera was set to fire as rapidly as possible and ran from before the beginning of the manoeuvre until its completion. The expiratory manoeuvre was completed three times with the camera firing at the same rate but with a staggered start. A sequence of photographs during inspiration was taken in exactly the same way. The volume of the trunk in each
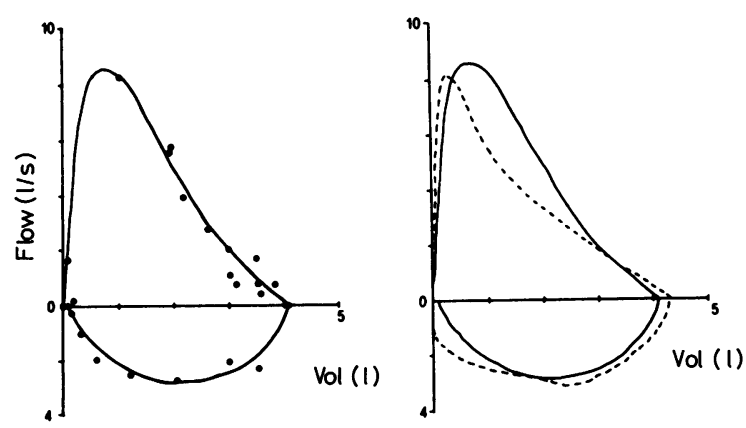

Fig 3 Left: Maximum expiratory flow volume loop derived from optically determined change in volume with time, plotted against optically determined volume in a normal subject. Right: The optically derived loop (solid line) superimposed on a loop derived from spirometric volume change (dotted line) plotted against spirometric volume in the same breaths. The measurement of vital capacity is underestimated by $5 \%$. The volume change of the trunk precedes air flow at the mouth. image was calculated and the time from the beginning of the manoeuvre determined from a digital clock, visible in the photograph. The incremental change in volume with time was plotted as flow against expired volume. Sixteen points were obtained on the expiratory limb and eight on the inspiratory limb. The optically derived flow volume loop was compared with the standard loop obtained from a pneumotachygraph and integrator (fig 3). The two loops have similar profiles but there is a $5 \%$ underestimate in the vital capacity. The change of optical chest volume precedes air flow, especially at the beginning of the manoeuvre. This is consistent with the compression of gas within the chest that occurs before expulsion. The discrepancy between trunk volume change and expired volume is a potential measure of alveolar pressure.

\section{Discussion}

The uses of optical techniques in medicine and industry have recently been reviewed by Duncan and Mair $^{3}$ and their application to the chest by Peacock et al.' This paper has reported an optical mapping procedure capable of describing the shape and motion of the thoracoabdominal wall in recumbent patients. It suggests that a single overhead camera can recover the coordinates of any point on the visible surface of the torso with accuracy. Evidence from work on a model torso indicates that it can assemble the information from a series of points on the surface and calculate the contained volume with a reproducibility of $\pm 26 \mathrm{ml}$ (SD) and a maximum error of $\pm 150 \mathrm{ml}$. Estimates from computed tomography scans of patients suggest that a single overhead camera can capture $97 \%$ of respired motion. Direct measurement on supine subjects shows that it can determine variations in respired volume within individuals with a mean accuracy of \pm 131 (SD 92) $\mathrm{ml}$.

There is, however, a systematic error of up to $\pm 10 \%$ in the measurement of respired volume, which varies from one individual to another and which is not apparent in measurements of the volume of physical objects. There are several possible explanations for this. The simplest is suggested by the computed tomography scans-namely, that there is a variation of up to $5 \%$ in the capture of respired motion from a single point of view. There could, however, be contributions from other sources, such as the uncertainty introduced by shadows on the surface of the visible segment-for example, between the breasts and in the supraclavicular fossae. The extent of this contribution is not clear but is probably small since we could find no correlation between the area of surface in shadow 
and the systematic error in the optical estimate of respired motion from one individual to another.

Although only half of the body surface is visible to the camera this can recover $97 \%$ of respiratory motion. This apparent paradox is because in the supine subject the back is restrained and motion occurs towards the point of observation. Moreover, the major shape and volume change occurs within the visible portion in normal subjects and in most patients. If an abnormality of motion is present beyond the visible boundary the patient can be repositioned so that this can be observed.

An important use of this technique may be the description of how each part of the thoracoabdominal wall moves with breathing, drawing attention to any temporal or spatial asymmetries or paradoxical motion. We have now examined many bedbound patients in this way, particularly tetraplegics. A valuable aspect, however, of the use of projected optical contours may be amplification of apparent motion of the surface so that the eye can appraise asymmetry and paradox more easily. These findings can be recorded with a video camera alone.
At present some stages of the analysis have to be done manually, up to 30 minutes being required for each image. The stripes of light that we use give information that is easy for the eye to appraise but is difficult to recognise automatically. In the future a different pattern - for example, with dots instead of stripes-may allow the process to become completely automatic.

We gratefully acknowledge the support of the Medical Research Council.

\section{References}

' Peacock AJ, Morgan MDL, Gourlay S, Turton C Denison DM. Optical mapping of the thoracoabdominal wall. Thorax 1984;39:93-100.

${ }^{2}$ Gourlay AR, Kaye G, Morgan MDL, Denison D, Peacock AJ. Analysis of an optical mapping technique for lung function studies. Computers in Biology and Medicine (in press).

${ }^{3}$ Duncan JP, Mair SG. Sculptured surfaces in engineering and medicine. Cambridge: Cambridge University Press, 1983. 